University of Nebraska - Lincoln

DigitalCommons@University of Nebraska - Lincoln

Faculty Publications in Food Science and Technology

2014

Development and evolution of risk assessment for food allergens

Rene W. R. Crevel

Joseph Baumert

Athanasia Baka

Geert Houben

André C. Knulst

See next page for additional authors

Follow this and additional works at: https://digitalcommons.unl.edu/foodsciefacpub

Part of the Allergy and Immunology Commons, and the Food Science Commons

This Article is brought to you for free and open access by the Food Science and Technology Department at DigitalCommons@University of Nebraska - Lincoln. It has been accepted for inclusion in Faculty Publications in Food Science and Technology by an authorized administrator of DigitalCommons@University of Nebraska Lincoln. 
Authors

Rene W. R. Crevel, Joseph Baumert, Athanasia Baka, Geert Houben, André C. Knulst, Astrid Kruizinga, Stefano Luccioli, Stephen L. Taylor, and Charlotte B. Madsen 
Invited Review

\title{
Development and evolution of risk assessment for food allergens
}

\author{
René W.R. Crevel ${ }^{\mathrm{a}}$, Joseph L. Baumert ${ }^{\mathrm{b}}$, Athanasia Baka ${ }^{\mathrm{c}, *}$, Geert F. Houben ${ }^{\mathrm{d}}$, André C. Knulst ${ }^{\mathrm{e}}$, \\ Astrid G. Kruizinga ${ }^{\mathrm{d}}$, Stefano Luccioli ${ }^{\mathrm{f}}$, Stephen L. Taylor ${ }^{\mathrm{b}}$, Charlotte B. Madsen ${ }^{\mathrm{g}}$
}

${ }^{a}$ Safety and Environmental Assurance Centre, Unilever, Colworth House, Sharnbrook, Bedford, UK

${ }^{\mathrm{b}}$ University of Nebraska, Lincoln, USA

${ }^{\mathrm{c}}$ International Life Sciences Institute - ILSI Europe, Brussels, Belgium

${ }^{\mathrm{d}}$ The Netherlands Organisation for Applied Scientific Research TNO, Zeist, The Netherlands

${ }^{\mathrm{e}}$ University Medical Centre Utrecht, Utrecht, The Netherlands

${ }^{\mathrm{f}}$ Center for Food Safety and Applied Nutrition, FDA, College Park, Maryland, USA

${ }^{g}$ Technical University of Denmark, Lyngby, Denmark

\section{A R T I C L E I N F O}

\section{Article history:}

Available online 6 February 2014

\section{Keywords:}

Food allergy

Public health

Risk assessment

Probabilistic modelling

Reference dose

Thresholds

\begin{abstract}
A B S T R A C T
The need to assess the risk from food allergens derives directly from the need to manage effectively this food safety hazard. Work spanning the last two decades dispelled the initial thinking that food allergens were so unique that the risk they posed was not amenable to established risk assessment approaches and methodologies. Food allergens possess some unique characteristics, which make a simple safety assessment approach based on the establishment of absolute population thresholds inadequate. Dose distribution modelling of MEDs permitted the quantification of the risk of reaction at the population level and has been readily integrated with consumption and contamination data through probabilistic risk assessment approaches to generate quantitative risk predictions. This paper discusses the strengths and limitations of this approach and identifies important data gaps, which affect the outcomes of these predictions. These include consumption patterns among allergic individuals, analytical techniques and their application, severity-dose relationships, and the impact of extraneous factors which alter an individual's physiology such as infection or exercise. Nevertheless, application of these models has provided valuable insights, leading to further refinements and generating testable hypotheses. Their application to estimate the risk posed by the concurrent consumption of two potentially contaminated foods illustrates their power.
\end{abstract}

(C) 2014 ILSI Europe. Published by Elsevier Ltd. All rights reserved.

\section{Contents}

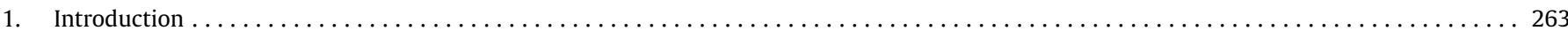

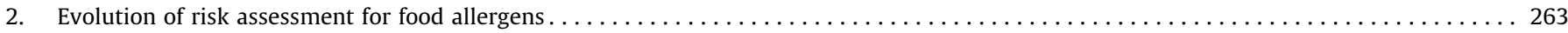

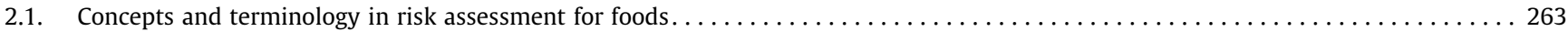

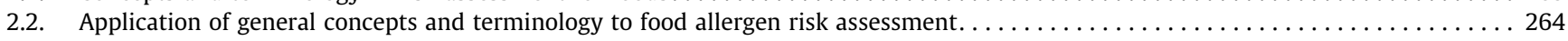

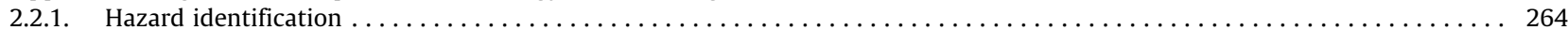

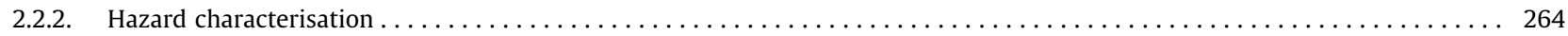

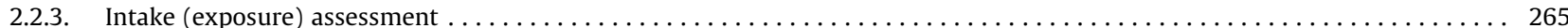

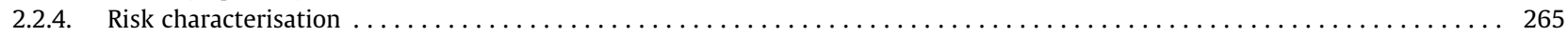

2.3. Development and evolution of practical approaches to the risk assessment for allergenic foods . . . . . . . . . . . . . . . . 265

3. Input parameters for the risk assessment: application, integration, uncertainties and limitations $\ldots \ldots \ldots \ldots \ldots$

3.1. Patient-derived data: uncertainties or other considerations in using controlled food challenge data in risk assessment . . . . . . . . 266

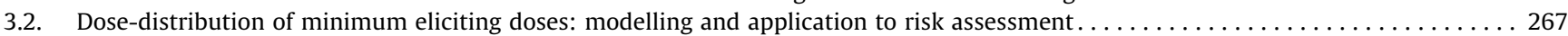

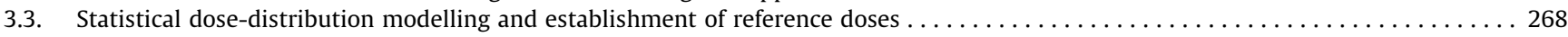

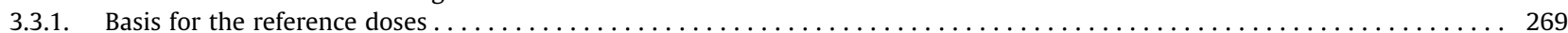

4. Intake assessment (consumption and contamination, quality and quantity of data, differences in behaviour, quality of analytical methods)... 270

\footnotetext{
* Corresponding author. Address: ILSI Europe a.i.s.b.l., Avenue E. Mounier 83, Box 6, 1200 Brussels, Belgium. Fax: +32 27620044.

E-mail address: publications@ilsieurope.be
} 


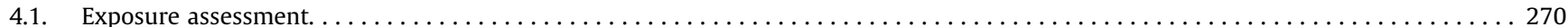

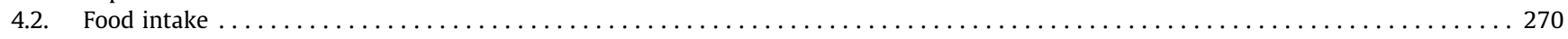

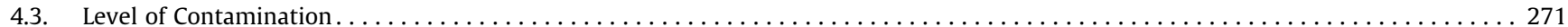

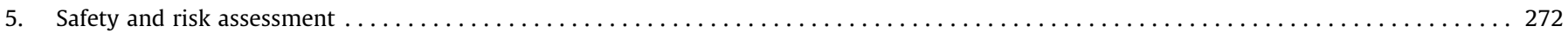

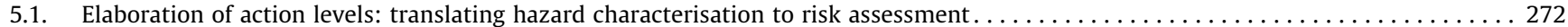

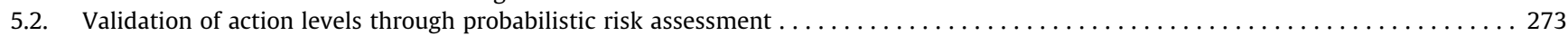

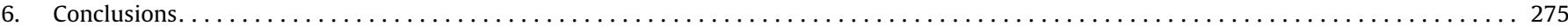

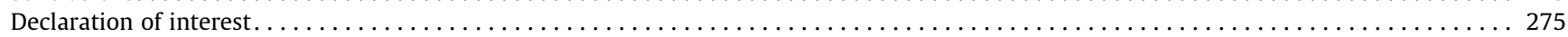

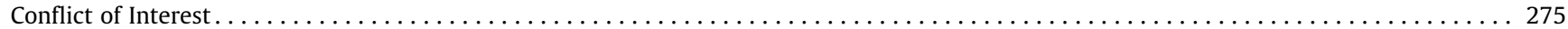

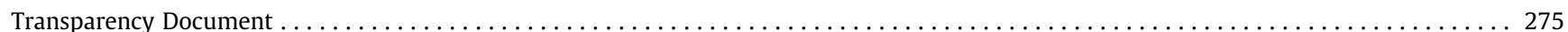

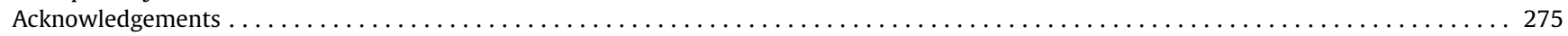

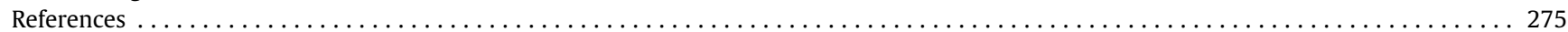

\section{Introduction}

The need to assess the risk from allergenic constituents or residues in manufactured food products arose very soon after food allergy was identified as a public health issue and mandatory food allergen source labelling as well as voluntary precautionary allergen statements on products ensued. It became apparent that failure to assess risk would lead to excessive warnings of unintended allergen presence on products and reduced food choices for people with food allergy. A pioneering piece of work demonstrated the feasibility of conducting allergen risk assessments when it showed to a standard higher than that required of hypoallergenic formulae that highly refined (N/RBD) peanut oil did not trigger reactions in people with peanut allergy, whereas crude peanut oil, which contained up to 100 times more protein, could do so (Hourihane et al., 1997a).

However, assessing the risk arising from the presence of allergenic constituents in products remained challenging. Initially the question was raised whether allergic reactions even obeyed classical toxicological principles, such as dose-response relationships. As data built up on reactivity within populations of people with food allergies (Taylor et al., 2004), it became clear that the range of reactivity to allergens is extremely wide, spanning at least 6 orders of magnitude, based on the results of controlled food challenge studies. The lack of knowledge about the distribution of this reactivity added to the perception generated by anecdotal case reports that most reactions occurred in response to extremely small amounts that would be difficult to measure, let alone manage in ordinary food manufacturing facilities.

Over the last decade or so, much progress has been made in filling a number of the data and knowledge gaps that prevented an adequate assessment of the risk. Food challenge data from studies designed to identify and characterise low dose reactors have become available in sufficient quantity to derive dose distributions for a large number of the more significant allergenic constituents. These data, on their own, would have been of much more limited value if the tools to analyse them had also not developed in parallel. Thus statistical modelling of dose distributions has become a widely accepted approach to characterising allergenic hazard (Bindslev-Jensen et al., 2002; Crevel et al., 2007). Similarly, probabilistic approaches to estimating the likely consequences of a particular pattern of allergen contamination are gaining currency (Spanjersberg et al., 2007), although gaps remain. These include inadequate data to characterise the hazard with confidence for some allergenic foods, but perhaps the most significant is understanding the background frequency of reactions that occur from day to day among allergic consumers and the associated pattern of severity. This background will in part be influenced by the food choices of allergic consumers (e.g. avoidance of certain food types, brands, etc.) and their acceptance of certain types of reaction (e.g. the milder variety). Inadequate knowledge about these food choices together with limited understanding of the frequency and range of product contamination point to an important data gap around the exposure component of the risk posed by food allergens.

Notwithstanding the remaining data and knowledge gaps, the progress to date, as well as initiatives such as the Allergen Bureau's of Australia and New Zealand VITAL initiative, demonstrated that allergen management could be placed on a sounder, evidencebased footing, based on robust risk assessment. This would improve the safety of allergic consumers, while providing the food industry with a clear set of standards towards which they could work. This paper presents the results of the work by the ILSIEurope Food Allergy Task Force's Expert Group and the discussions with stakeholders concerning risk assessment of allergenic foods.

\section{Evolution of risk assessment for food allergens}

Allergens in food pose a health risk to humans who may become sensitised or are already sensitised to these proteins. Terminologies and methodologies for food risk and safety assessment and management developed and evolved largely in response to the threats to public health from chemical or microbiological contaminants that may be present in food. For a long time, it was questioned whether classical toxicological risk assessment principles could be applied to allergy and allergenic foods. However, with better understanding of reactions to allergens, together with increasing volumes and quality of data, it is now widely accepted that the broad principles and approaches of chemical toxicology risk assessment can be applied to food allergens (Madsen et al., 2009; Spanjersberg et al., 2007, 2010), as can the same terminologies and methodologies. This section describes the concepts and terminology in (mainly chemical) food safety and risk assessment and their applicability to allergens. In addition, practical approaches that have been developed for food allergen risk assessment are described.

\subsection{Concepts and terminology in risk assessment for foods}

Clarity of thought and communication among risk assessors and managers are critical in ensuring good management of risk and much effort has been expended on international harmonisation of terminologies. In this paper, terminology is based on definitions set out by the INTERNATIONAL PROGRAMME ON CHEMICAL SAFETY (IPCS) in 2009 in their "Alphabetical list of selected key generic terms in hazard and risk assessment and their definitions" (INTERNATIONAL PROGRAMME ON CHEMICAL SAFETY HARMONIZATION PROJECT). This terminology has been harmonised across the different disciplines covered by the Codex Alimentarius Commission and thus also applies to microbiological risk assessment 
for foods, to which risk assessment for allergens also bears a number of similarities (Codex Alimentarius Commission, 1999b).

Allergens, like chemicals and microorganisms, may pose a risk to human health due to their inherent properties, otherwise known as the hazard and specifically defined as: an inherent property of an agent or situation having the potential to cause adverse effects when an organism, system, or (sub)population is exposed to that agent. The probability of an adverse effect in an organism, system, or (sub)population caused under specified circumstances by exposure to an agent is called risk. A concept closely linked to risk is safety. Safety may have various (legal or societal) definitions, of which one is the practical certainty that significant adverse effects will not result from exposure to an agent under defined circumstances. It can also be represented as the reciprocal of risk and may also be perceived as a risk that society accepts.

Risk analysis is the overall process for controlling situations where an organism, system, or (sub)population could be exposed to a hazard. The risk analysis process consists of three components: risk assessment, risk management, and risk communication.

Risk assessment describes the process through which is estimated the risk to a given target organism, system, or (sub)population, including the identification of attendant uncertainties, following exposure to a particular agent, taking into account the inherent characteristics of the agent of concern as well as the characteristics of the specific target system including the (sub)population. It includes four steps: hazard identification, hazard characterization, exposure assessment, and risk characterization. Hazard identification can refer to the determination that a substance with hazardous properties is present, but is more generally meant as the identification of the type and nature of the adverse effects that an agent can cause and as such its inherent capacity to cause these in an organism, system, or (sub)population. In hazard characterization, a qualitative and, wherever possible, quantitative description of the inherent property of an agent or situation having the potential to cause adverse effects is made, together with its relationship to dose, where possible. Hazard identification and hazard characterization together constitute hazard assessment.

The hazard assessment findings can be used to derive safe levels of exposure, for instance through the elaboration of a Reference Dose, a term which IPCS uses to describe the daily dose that is likely to be without deleterious effect even if continued exposure occurs over a lifetime. An example of a Reference Dose is the Acceptable Daily Intake (ADI): the estimated maximum amount of an agent to which individuals in a (sub)population may be exposed daily over their lifetimes without appreciable health risk. Often, a Reference Dose or ADI is used as a basis for calculating maximum tolerable levels of substances in food. Many food safety standards have been set through this process of assessing hazard data and establishing a Reference Dose and a maximum level for food.

Since dose is a parameter critical to the risk posed by a substance, exposure assessment forms an essential part of determining whether the hazardous properties of a substance will translate to adverse health effects. Results of an exposure assessment can be directly compared with an established assessment of the hazard information that resulted in a Reference Dose or established maximum level in food. An exposure at or below the Reference Dose or maximum level is assumed to be safe. In cases where past, current or expected exposure is in excess of a Reference Dose or maximum level, or in cases where a reference Dose or maximum level has not been established, a risk characterization may be performed to calculate or estimate the risk. The risk characterization may be defined as the qualitative and, wherever possible, semi-quantitative or quantitative determination, including attendant uncertainties, of the probability that known and potential adverse effects of an agent will occur in a given organism, system, or (sub)population, under defined exposure conditions.

A risk assessment may lead to the conclusion that the risk needs to be mitigated, in which case a risk management phase will follow, together with communication of that risk, as appropriate.

\subsection{Application of general concepts and terminology to food allergen risk assessment}

Assessing the risk from allergens does not fundamentally differ from assessing the risk from chemical substances or microbiological agents, although there are clearly distinct features associated with each. Thus some aspects of allergen risk assessment resemble those pertaining to chemical substances, while others share parallels with microbiological agents. This may be best illustrated by considering each of the steps.

\subsubsection{Hazard identification}

For foods that are already commonly consumed, hazard identification occurs retrospectively through case reports, rather than prospectively, through predictive toxicological tests. Thus a food is identified as allergenic, because individuals are reported to react to it in a manner consistent with allergic reactions mediated by IgE antibodies. Hazard identification is then ultimately completed by demonstration of IgE binding to individual proteins in the food and confirmatory tests including clinical controlled oral challenges in affected individuals. Therefore, in allergies to commonly consumed foods, the hazard of concern is the ability to elicit reactions in sensitised individuals, rather than the ability to sensitise in the first instance. Currently, unlike most food chemicals, there are no validated animal models to assess the toxicity of food allergens; thus, only reactions in sensitised individuals can be considered adequate confirmation that a food is an allergenic hazard in humans. Hazard identification therefore relies completely on human data from previously exposed and sensitised individuals, resembling microbiological hazard identification, which relies principally on epidemiological and surveillance data rather than prospective studies in animals.

\subsubsection{Hazard characterisation}

Hazard characterisation for allergens arguably possesses advantages compared to characterisation of both chemical and microbiological hazards. As it relies on human data, animal to man extrapolation of the results of toxicological studies and consideration of various other qualitative and quantitative uncertainties associated with non-human toxicity data are unnecessary. This constitutes a distinct advantage for food allergen risk assessment compared to traditional chemical risk assessment. However, the need for human data also imposes ethical and practical constraints that limit both the amount and type of data that can be generated. Challenge studies rely on volunteer participants who can only be tested a limited number of times and furthermore may not be fully representative of the whole population allergic to a food. The availability of suitable clinics and trained personnel, as well as the prevalence of allergy to a particular food further limit the numbers that can be tested. Detailed assessment of the relationship between the dose and the response must comply with the requirements not to harm the individuals under investigation. Because of the risks posed and the ethical considerations, deliberate administration of microbiological agents to investigate dose response relationships is rare, whereas with food allergens it can be included as part of the diagnostic protocol since the information obtained is useful to the individuals involved as well as from a population perspective. Thus food allergen hazard can be much more accurately characterised. Notwithstanding the limitations inherent in human studies, increasing amounts of data of ever better quality continue to 
become available on the relationship between minimum eliciting dose and frequency of reaction in the population allergic to a number of priority allergens. These data demonstrate also that thresholds for the elicitation of allergic effects by food allergens exist at an individual level and thus also at a population level. Depending on the risk management questions and the goal, focus and approach of the risk assessment, uncertainty factors may be applied. Probabilistic modelling (see subsection 5.1), takes account of the uncertainty and variability associated with each input variable and therefore negates the need to apply, often arbitrary, uncertainty factors to the risk assessment output. This is another potential advantage compared to traditional chemical risk assessments.

\subsubsection{Intake (exposure) assessment}

In principle, this step does not differ in any significant way from that required for chemical risk assessment. However, the exposure metric for food allergens is the amount consumed on a single eating occasion, or within a relatively short period of time, rather than long-term exposure as with chemical exposure assessment. The exposure assessment for allergens therefore resembles much more closely microbiological exposure assessment inasmuch as the focus is on short-term acute effects rather than long-term effects (Codex Alimentarius Commission, 1999b). Yet, important differences remain. These include the need to take account of the growth of microorganisms at different stages and the fact that the risk assessment for food allergens, unlike for chemical contaminants or microorganisms in general, focusses on a relatively small subpopulation, a high proportion of which are aware of the presence of specific hazards in food that may pose a risk to them: allergens. Their patterns of food consumption might therefore differ from that of the general population. This could affect their total exposure and therefore risk, either decreasing it or increasing it depending on the product. It may be assumed, but this is yet to be confirmed, that differences in food choice will be larger than differences in the amounts of certain food products consumed by allergic and non-allergic users. In other words, allergic and non-allergic consumers of a food will consume a broadly similar amount on each eating occasion. To the best of our knowledge, no structured broad (nationwide) studies have been conducted that address this factor, although the MIRABEL study in France will begin to yield insights in the near future (Nguyen-Grosjean, 2013). Also lacking are any structured surveys into the distribution of specific allergens present by cross-contact in the general food supply, a situation made worse by the limitations of current tools. This hampers the translation of food consumption data into allergen intake data.

\subsubsection{Risk characterisation}

The final step in the allergen risk assessment process also needs to be applied in a manner adapted to the limitations in knowledge and data, as well as to the different circumstances associated with allergenicity discussed above. A risk assessment needs to be attuned to the ultimate purpose of the assessment, which needs to be specified at the problem formulation stage. For allergens in food, this risk assessment can have two major purposes: (1) the characterisation of a risk associated with a defined (range of) level(s) of (residual) allergen in a food product and (2) the establishment of (safe) limit levels for (residual) allergens in food. These are addressed in more detail in the next sections.

\subsection{Development and evolution of practical approaches to the risk assessment for allergenic foods}

Many foods have the potential to provoke allergic reactions in sensitized individuals (Hefle et al., 1996), but a relatively limited number has been identified as priority allergenic foods based upon perceptions of the prevalence, severity and potency of those foods as allergens (Codex Alimentariu Commission, 1999; EFSA, 2004; Gendel, 2012). In the EU, the list of priority allergenic foods comprises peanut, milk, eggs, fish, crustacean shellfish, molluscan shellfish, tree nuts, soybeans, cereal sources of gluten (wheat, rye, barley and related grains), lupine, celery, sesame seed and mustard. Bjorksten et al. proposed a set of consistent scientific and clinical criteria to identify the most appropriate priority allergenic foods based upon defined prevalence, severity and potency criteria (Bjorksten et al., 2008). While this report focusses on the priority allergens already identified on the EU list, the approaches described should be applicable for foods on other lists, where sufficient data exist.

Multiple approaches have been considered to characterise the hazard and assess the risk from residues of allergenic foods that might be present in other foods. The main focus has been on the risk posed by the potential unintended and variable presence of those allergenic residues that, not being ingredients, are not subject to mandatory declaration on product labels. However, similar approaches could be adapted for allergenic ingredients used at very low levels. The use of clinical oral food challenge data to develop dose-distribution models for populations with specific food allergies was first attempted by Bindslev-Jensen et al. (2002). In a 2007 workshop organized by EuroPrevall, the U.K. Food Standards Agency, and ILSI-Europe, three main, non-mutually exclusive risk assessment approaches were identified: (1) safety assessment using the No Observed Adverse Effect Level (NOAEL) and/or the Lowest Observed Adverse Effect Level (LOAEL) with application of uncertainty factors, (2) the Benchmark Dose and Margin of Exposure (MoE) approach, and (3) the use of probabilistic models (Madsen et al., 2009). The workshop concluded that the MoE and probabilistic approaches have merit because they do not rely upon low-dose extrapolations from dose-distributions derived from clinical challenges with their inherent uncertainties. They also make use of the whole dataset, in contrast to the safety assessment approach which relies on a single data point, making it also highly dependent on the quality of study design. Probabilistic modelling can be used in conjunction with establishment of Benchmark Doses. In the U.S., the Threshold Working Group of the Food \& Drug Administration independently considered multiple approaches to the risk assessment of residues of allergenic foods including a statutorily derived approach, a limit of detection approach, the deterministic safety assessment approach with applied uncertainty factors, and the quantitative risk assessment (which includes probabilistic modelling) approach (Threshold Working Group, 2008). This U.S. FDA group also concluded that the quantitative risk assessment approach provided the strongest and most transparent scientific approach to establish management or regulatory thresholds for allergenic foods. This group also noted that this approach was the most data-intensive and that insufficient data were likely available to use this approach in a robust manner. The European group also indicated that larger volumes of data on minimum eliciting doses were needed to allow effective use of the probabilistic modelling approach (Madsen et al., 2009).

In the absence of guidance on agreed limits for allergenic residues, many global food companies have used the limit of detection as a default criterion, making it a de facto action level. As noted by U.S. FDA, the limit of detection is not necessarily selected on the basis of the magnitude of the remaining allergenic hazard (Threshold Working Group, 2008) and in practice, can be very conservative. This is especially so when using swabs to verify cleaning as the methods are highly sensitive as compared to the rate of transfer from surfaces to products, dilution factors and other considerations.

In Australia, the Food \& Grocery Council (trade association) established The Allergen Bureau of Australia \& New Zealand in an attempt to improve the practice of allergen management and, in particular curtail the widespread use of advisory or precautionary 
labelling. The Allergen Bureau developed a practical approach to allergen risk assessment in 2007 known as the VITAL (Voluntary Incidental Trace Allergen Labelling) programme. An essential part of the VITAL programme was the establishment of an allergen grid containing Action Levels to guide the use of precautionary labelling. The grid levels were established using a deterministic safety assessment approach, based LOAELs cited in the U.S. FDA compilation (Threshold Working Group, 2008) and included a 10-fold uncertainty factor to reflect the limitations of the available clinical data, including the use of LOAELs rather than NOAELs.

The experiences of the Allergen Bureau offer useful lessons. In the 2007 version of VITAL, three Action Levels were established to guide food industry labelling decisions: Green (low risk; no precautionary labelling); Yellow (possible risk; precautionary "may be present: \#\#\#\#" labelling recommended); Red (higher risk; definitive "contains \#\#\#\#" labelling recommended). Initially, most of the green levels were set at $<2$ ppm of protein from the allergenic food (exceptions: fish, milk, soy, gluten). While the VITAL programme recognised that individual and population thresholds were actually doses of allergenic protein rather than concentrations, they expressed the Action Levels in terms of ppm of total protein from the allergenic food since allergen assays give results as concentrations. However, analytical methods differ in the expression of their results (e.g. ppm whole food, ppm specific protein, etc.) as a result of which conversion factors are needed to apply results. Furthermore, the Action Levels were based upon a $5 \mathrm{~g}$ (approx. one teaspoon) serving size on the assumption that an allergic person would perceive some reaction before they proceeded to consume more of the implicated food and would therefore be protected. Clearly, many foods are consumed in quantities well above $5 \mathrm{~g}$ and can be consumed in their entirety before a reaction becomes apparent. There is therefore a need to consider consumption patterns and amounts as part of the risk assessment process. This can be accomplished using quantitative risk assessment as outlined further in this report. The VITAL program was revised in 2011 to incorporate consideration of the availability of more clinical data and the range of consumption levels for various foods.

\section{Input parameters for the risk assessment: application, integration, uncertainties and limitations}

\subsection{Patient-derived data: uncertainties or other considerations in using controlled food challenge data in risk assessment}

Data derived from patients in controlled clinical challenges form a cornerstone of the risk assessment since they characterise the hazard associated with a given allergenic food. While this source of data offers a number of advantages for the risk assessment (e.g. no species to species extrapolation, good evaluation of individual variability), it also possesses some notable limitations. As noted above, clinical challenges are controlled in various ways relating to general health status, medication use, and others that differ from the wider range of circumstances encountered in a community setting.

Thus, a legitimate question is the extent to which these results apply when the individual encounters the allergenic food in the wider community. Review of the literature reveals a range of factors associated with the host as well as with the food which could affect a person's clinical reactivity on any given occasion and therefore potentially their observed minimum eliciting dose (Table 1).

An attempt was made to analyse available clinical literature to identify factors that could influence MED dose distribution curves and distinguish those originating at the individual level population levels, as well as incorporating those associated with the foods, and
Table 1

Factors impacting clinical reactivity and population distribution of minimum eliciting doses.

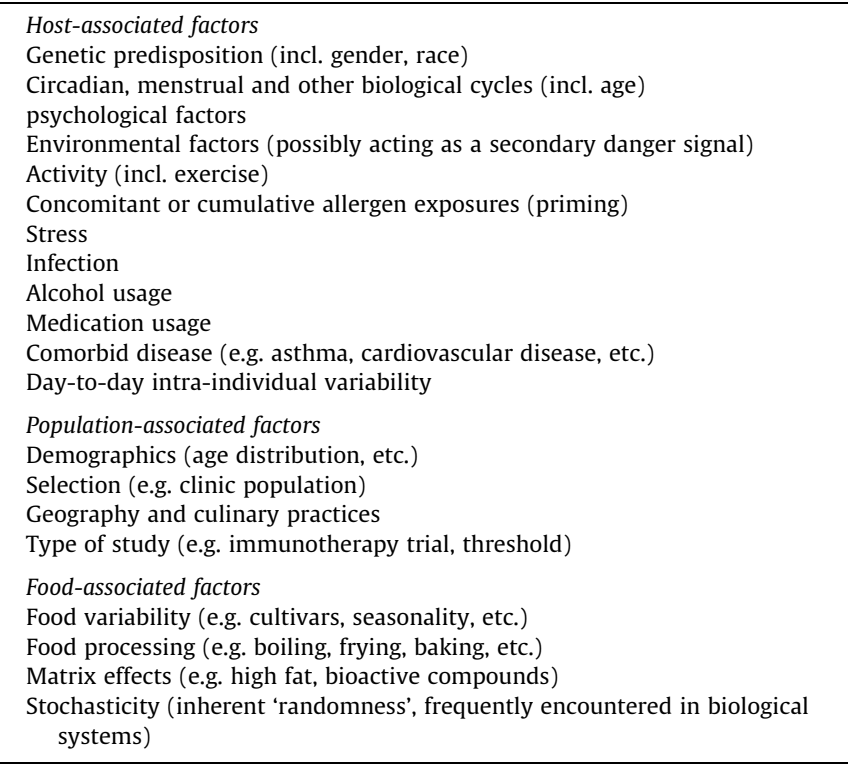

assess their possible quantitative impact. However, data are extremely scarce in this field beyond anecdotal evidence and quantitative estimates of the influence of any factor are non-existent for practical purposes. Many of these factors (i.e., genetic predisposition and host-environmental interactions) would be expected to induce minimal variability in estimated threshold doses.

Other sources of variability such as biological cycles, psychological factors, stress, and concomitant allergen exposures (e.g. seasonality related to pollen) are likely to already be incorporated implicitly into the threshold data sets because attempts are not made to control these factors during clinical challenges. Stochasticity is also likely to be incorporated into existing data sets especially those data sets involving larger and more unselected groups of subjects. Food-associated factors, especially food processing and matrix effects, are likely to be important factors that merit consideration, although their influence will differ by food product. They are discussed later in this report. To some degree, the argument can be made that the existing clinical challenge data predominantly reflects the worst-case scenario by incorporating a mildly processed form of the food into a rather simple matrix, which makes it highly bioavailable. Admittedly, this aspect merits additional clinical investigation and highlights the importance of fully characterising challenge materials, as well as ensuring that the allergens are presented in a form that is representative of what is likely to be encountered in free-living situations.

That analysis leaves activity (especially exercise), infection, alcohol usage, and medication usage as sources of uncertainty that differ between the community and clinical settings. The quantitative impact of these factors on threshold doses and therefore on the probability of reactions has not been well investigated but is acknowledged to be potentially important. To some degree, they can ideally be addressed in clinical guidance where food-allergic patients are given advice to exercise greater care and vigilance with avoidance diets during times of infection, use of particular medications, or around periods of intense physical activity.

Variability attributable to the selection of the populations investigated in challenge studies has already been shown to be potentially significant (Taylor et al., 2009), but data have not been published on systematic studies in this area. The practice in many clinics to exclude from challenges individuals who report prior 
severe (anaphylactic) reactions may reduce the representation of individuals with greater sensitivity, and therefore lower MEDs, although this remains unproven and the quantitative impact has not been estimated (e.g. by considering the proportion of individuals excluded). However, the more profound impact will result from the exclusion of less sensitive individuals who are able to manage their allergies without recourse to clinical help. A consequence of their ability to manage is that they will often not be recruited into studies since they will not be known to the relevant health services. Furthermore, they will probably not be motivated to participate in a procedure which requires a significant investment of their time for no clear benefit. Thus, the overall result of population selection biases will tend to make estimates of population eliciting doses rather conservative.

\subsection{Dose-distribution of minimum eliciting doses: modelling and application to risk assessment}

Initial approaches to establish safe limits for allergenic residues focussed on a deterministic approach based on identifying a NOAEL for the allergic population. Empirically, clinical investigators have known for many years that, once sensitized, some food-allergic individuals could react adversely to ingestion of small quantities of an allergenic food (Gern et al., 1991; Malmheden-Yman, 2004). However, no one knew exactly how small the amounts needed to be. Blinded oral food challenges were (and remain) the only unequivocal means for the diagnosis of food allergies but the procedure itself has evolved and adapted to new requirements. Early investigations started at doses of $500 \mathrm{mg}$ of food (Bindslev-Jensen et al., 2004; Bock et al., 1988) and resulted in a substantial proportion ( $20 \%$ or more) of patients reacting at the first dose, and therefore could not establish a NOAEL (Sicherer et al., 2000). As challenge procedures were refined and improved, particularly with the inclusion of very low starting doses, it rapidly became apparent firstly that the ultimate population NOAEL would be elusive, but more importantly that it might prove so low that it would be unsuitable for risk management decisions and finally that it would make very poor use of the growing body of data. These observations, together with the growing volume of data on low dose food challenges, thus spurred consideration of alternatives to the classical chemical safety assessment.

The more widespread use of low dose oral challenges in clinical practice has confirmed that each food-allergic individual has a threshold dose below which they will not experience an adverse reaction upon ingestion of the allergenic food (Hourihane et al., 1997b; Taylor et al., 2002). Thus, double-blind, placebo-controlled food challenge (DBPCFC) trials in clinical settings provide human data on food-allergic individuals that can be used for risk assessment purposes.

Before evaluating the clinical data for possible use for risk assessment purposes, several definitions must be established starting with a definition for the term, threshold. Clinically, the threshold dose is operationally defined as the lowest dose capable of eliciting an allergic reaction and can also be termed the LOAEL or the minimum eliciting dose (MED) (Crevel et al., 2007). However, for risk assessment purposes and in parallel with general toxicological practice, the NOAEL, the highest amount of the allergenic food which will not cause a reaction in a particular individual allergic to that food, is also important. In the existing clinical literature from low-dose oral challenge trials, individual LOAELs are reported but NOAELs are often not, but can be discerned if the dosing scheme is provided. The precision of both the LOAEL and the NOAEL are dependent on study design, which therefore also contributes to uncertainty.

To determine an individual NOAEL or LOAEL, agreement must be reached on what constitutes an allergic reaction. In the existing clinical literature from low-dose challenge trials, both subjective responses and objective reactions have been reported. Objective reactions are responses apparent to an external observer, such as urticaria, erythema, and vomiting. Subjective responses, such as palatal itching, and nausea, are, by definition, not apparent to external observers, such as clinical staff, and their recording is dependent upon the verbal comments of the patients, often in response to questioning from staff. Because of their nature, objective reactions can be considered generally a more reliable indication of a specific response to a food. In clinical settings, objective symptoms can be confirmed to occur and their reproducibility readily assessed. Furthermore, use of an objective marker reduces potential uncertainty in interpretation of symptoms when different populations are analysed together (e.g. infants and adults or older children). In low-dose challenge trials, severe reactions are not typically encountered. In contrast, subjective symptoms such as non-cooperation (in children particularly) or itch are easily elicited but difficult to quantify on a consistent basis between clinical investigators and can be difficult to attribute unequivocally to a specific response to the food. While subjective responses provide valuable information and should be recorded, consensus was reached that the NOAEL and LOAEL should be established on the basis of the doses provoking the first objective reactions in DBPCFC trials, with abdominal pain in infants and young children as the main exception.

Current dose distribution models are asymptotic and therefore do not define a dose at which no individual is predicted to react. However, operationally, the population threshold can be defined as the largest amount of the allergenic food which will not cause a reaction when tested experimentally in a defined population of allergic individuals e.g. all tested peanut-allergic individuals (the population NOAEL). This definition relies upon the presumption that a representative group can be identified within the global population of individuals allergic to a specific food and tested clinically with oral challenges. The degree to which the tested group represents the overall population with a specific food allergy is a source of potential uncertainty.

While population eliciting doses are likely to be conservative estimates for reasons discussed previously, concern has been voiced that some of the most highly sensitive individuals (as defined by dose) might have been excluded from challenge studies. However this concern equates sensitivity with a history of severe reactions, a link which still remains to be proven. In a retrospective study of peanut thresholds, Taylor et al. (2010) observed no difference between those of peanut-allergic patients who reported histories of severe reactions and those of patients whose previous reactions had been less severe. Another recent challenge study found no consistent relationship between the dose at which reactions occurred and the severity of the reaction (RolinckWerninghaus et al., 2012). Considerable variation exists among individuals with a specific food allergy with respect to their individual NOAELs and LOAELs (Crevel et al., 2007). In an examination of individual thresholds among 450 peanut-allergic individuals, the range of individual LOAELs spanned five orders of magnitude from $0.1 \mathrm{mg}$ up to $2.5 \mathrm{~g}$ of peanut protein or $0.4 \mathrm{mg}$ to $10 \mathrm{~g}$ of whole peanut (Taylor et al., 2010). Given that such a wide range of individual LOAELs exist, the selection of patients for oral challenge trials used to establish population thresholds becomes quite important, as is an accurate description of the allergological status of the participants. Thus the data should be examined carefully to ascertain whether the selected patients are representative or skewed in favour of selection of patients likely to react to lower doses. Ideally, it should be possible to map the characteristics of the challenged individuals onto the overall population allergic to the food under study and thereby identify biases that might need to be corrected, or at least acknowledged in the risk assessment 
conclusions. However, comparative studies have not been performed between patients from different geographical areas, patients of different ages, the same patients in different seasons (e.g. pollen season), or patients with differing symptoms. Instead, existing evaluations have used data from patients from all groups. In practice, the expertise required to perform challenge studies means that they are generally performed in tertiary care reference clinics, where the overall patient populations are more likely to have multiple allergies and a history of at least one severe reaction. However the selection of individuals for challenge studies may not necessarily mirror the clinic population as a whole.

When examining clinical information for the purpose of establishing population thresholds, variations in clinical challenge protocols must be considered. A consensus protocol for low-dose clinical challenges has been developed (Taylor et al., 2004) and close variants applied to large-scale studies (Crevel et al., 2008). Widespread consensus has been achieved on various protocol elements such as withholding of certain medications before and during challenges, time intervals between doses, use of placebocontrolled crossover designs, use of objective symptoms (or abdominal pain in infants and young children) as the criterion for stopping challenges, and fasting period before challenges. However, comparison of data from clinics which used different protocols must take into account various other factors must be considered such as the nature of the challenge materials, the matrix for blinding of challenges, and dosage schemes (Crevel et al., 2008).

Challenge materials are a critical variable in challenge studies and should be fully characterised, as in any safety study, both with regard to the active material (the food to be blinded) and the actual dose present in the matrix. The outcome of challenges can be affected by the form of the allergenic food, the vehicle, or the type of processing used in the preparation, which may, for instance, alter the proportions of different allergenic proteins (Ballmer-Weber et al., 2007). The form of the allergenic food (e.g. peanut flour vs. ground roasted peanuts vs. peanut butter or soya flour v soya milk) and the extent to which it is processed varies between challenge protocols but may not be important in the case of foods such as peanuts where allergens are thought to be heat-stable. With milk and egg however, challenges should ideally use less processed forms of food such as pasteurized, spray-dried or even raw, where possible. Recent clinical studies have documented that many milkand egg-allergic patients become tolerant of baked milk or egg before they develop a tolerance for these foods in forms that are subjected to lesser degrees of heat processing, and this is reflected in increased individual thresholds (Lemon-Mule et al., 2008; Nowak-Wegrzyn et al., 2008). The use of raw or mildly processed foods thus appears to represent a worst-case situation relative to individual threshold doses but the materials used need to reflect those used in commercial food production since they are the principal subject of the risk assessment. The outcome of challenges may also be dependent upon the composition of the vehicle used for challenges such as the level of fat e.g. chocolate vs. other vehicles (Grimshaw et al., 2003; Cochrane et al., 2012).

The dosage schemes used in clinical challenge protocols also vary. However, a statistical approach called interval-censoring survival analysis has been developed that adjusts to different dosing schemes and is likely to remove this factor from consideration, although results can still be influenced if a large proportion of the data are not interval-censored (e.g. first dose reactors) (Taylor et al., 2009).

Beyond clinical protocol variables, the results of DBPCFCs must be evaluated on the basis of several other factors. For example, challenge doses are typically administered at intervals of 20-30 min that probably do not allow for complete assimilation of one challenge dose before the next dose is administered. It is therefore not possible to know with certainty whether a reaction occurred in response to the last dose administered, a delayed response to a prior dose or to the cumulative dose ingested. Challenge results can thus be analysed in terms of both discrete and cumulative doses. The nature of the population selected for challenge is another variable, which has already been discussed. However, separate analysis of different (sub)populations (e.g. children vs. adults) may provide valuable insights, although only where a sufficient volume of data make it statistically valid. Geographical, ethnic or other differences might also occur between sub-populations with a particular food allergy but clinical data to establish a basis for such differences are still very limited outside the well-known influence of pollen sensitisation on certain food allergies. Potential resolution of certain food allergies such as milk and egg in infants and children (Sampson, 1996) must also be taken into account in evaluating data from such populations.

\subsection{Statistical dose-distribution modelling and establishment of reference doses}

While the determination of individual threshold doses for foodallergic patients is not currently a common clinical practice, data from low-dose DBPCFC trials have become increasingly available in recent years. In the published clinical literature, individual threshold data can be found from three different types of studies - diagnostic series, threshold studies and immunotherapy trials (Taylor et al., 2009; Skripak et al., 2008; Clark et al., 2009). Allergy clinics are also likely to have potentially useful but unpublished individual threshold data. From the available clinical information, statistical dose-distribution modelling of individual NOAELs and LOAELs can be used to derive information useful in the establishment of a population threshold.

Published clinical data relevant to thresholds are typically not presented in a consistent format. Some of the key differences between studies are described elsewhere in this report. A first step in analysing these data is to normalize them to allow inclusion of data from multiple centres and studies. For the purposes of this report, threshold data are normalized to total protein content from the allergenic source. The use of total protein from the allergenic source is an appropriate unit of measure because food allergens are proteins, and represents a pragmatic solution, since data, such as detailed characterisation of the foods used in challenges, are not generally available to perform a more sophisticated normalization Since different forms (e.g. liquid, solid, etc.) of an allergenic food can be used in various studies, normalizing on the basis of total protein from the source corrects for any differences that might exist on a food weight basis. Furthermore, analytical methods for allergenic foods are often based on the total protein content allowing intake assessment to be matched to reactive doses.

The statistical models using data from the clinical challenge studies allow the derivation of an eliciting dose (ED), where EDp refers to the dose of total protein from the allergenic food that is predicted to produce a response in $\mathrm{p} \%$ of the allergic population (Crevel et al., 2007). This approach enables the identification of a dose of an allergenic food protein (EDp) at or below which a defined proportion of the allergic population would be likely to react to but, importantly, does not identify a dose below which no allergic individual would react. The reference doses described in this report (Table 2) were derived from clinical data obtained from DBPCFC trials except in the case of infants and young children where blinding is considered not necessary. The endpoints of individual challenges were the provocation of objective symptoms with the exception of abdominal pain in infants and young children. The data were modelled on the basis of both discrete and cumulative doses. 
Table 2

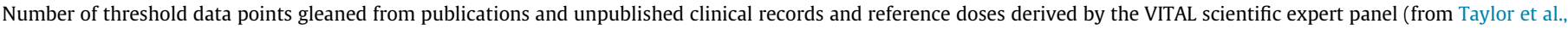
2014).

\begin{tabular}{|c|c|c|c|c|c|c|}
\hline Allergen & Total with Objective Symptoms & Right Censored $^{a}$ & Left Censored $^{\mathrm{b}}$ & Population & Basis of Reference Dose & Reference Dose (mg total protein) \\
\hline Peanut & 750 & 132 & 30 & Children and Adults & ED01 & $0.2 \mathrm{mg}$ \\
\hline Milk & 351 & 19 & 59 & Children and Adults & ED01 & $0.1 \mathrm{mg}$ \\
\hline Egg & 206 & 33 & 24 & Children and Adults & ED01 & $0.03 \mathrm{mg}$ \\
\hline Hazelnut & 202 & 67 & 4 & Children and Adults & ED01 & $0.1 \mathrm{mg}$ \\
\hline Soybean & 80 & 28 & 6 & Children and Adults & LCI ED05 & $1.0 \mathrm{mg}$ \\
\hline Wheat & 40 & 1 & 5 & Children and Adults & LCI ED05 & $1.0 \mathrm{mg}$ \\
\hline Cashew & 31 & 16 & 1 & Children & Hazelnut (ED01) & $0.1 \mathrm{mg}$ \\
\hline Mustard & 33 & 10 & 2 & Children and Adults & LCI ED05 & $0.05 \mathrm{mg}$ \\
\hline Lupin & 24 & 7 & 2 & Children and Adults & LCI ED05 & $4.0 \mathrm{mg}$ \\
\hline Sesame & 21 & 1 & 2 & Children and Adults & LCI ED05 & $0.2 \mathrm{mg}$ \\
\hline Shrimp & 48 & 26 & 0 & Adults & LCI ED05 & $10 \mathrm{mg}$ \\
\hline Celery & 39 & 4 & 15 & Children and Adults & & NR \\
\hline Fish & 19 & 2 & 6 & Children and Adults & LCI ED05 & $0.1 \mathrm{mg}$ (provisional) \\
\hline
\end{tabular}

NR: No recommendation.

a Number of right-censored subjects (NOAEL = highest challenge dose; LOAEL set to infinity).

b Number of left-censored subjects (NOAEL set at zero; LOAEL = lowest challenge dose).

Where possible, individual data were gleaned from published clinical research. Publications were selected using criteria outlined previously in particular focusing on DBPCFC trials involving use of low doses. Additional unpublished data were obtained from several clinics in the Netherlands and Berlin, Germany (gathered by TNO) and from partially completed threshold research studies sponsored by the Food Allergy Research \& Resource Program of the University of Nebraska (USA). The NOAEL and LOAEL for objective responses were recorded for each subject in units of $\mathrm{mg}$ of total protein from the allergenic food. For each subject, the true threshold dose lies, by definition, between the NOAEL and LOAEL doses. Individual threshold data were analysed using interval-censoring survival analysis (Taylor et al., 2009). The data were fitted to the parametric models using the SAS LIFEREG procedure (SAS v9.1) as described by Taylor et al. (2009). Several parametric models (log normal, log logistic, Weibull) were used to fit the individual threshold data for each allergenic food. The totality of evidence from all three models was considered in deriving the appropriate EDp, as well as the overall goodness of those fits, rather than attempting to select the single apparent best fit. This approach was deemed more robust since there is currently no biological rationale to prefer one parametric model over another. The outcome of the dosedistribution modelling was the derivation of a reference dose in units of $\mathrm{mg}$ of total protein from the allergenic food.

\subsubsection{Basis for the reference doses}

Reference doses represent a judgement about accepted risk. Where the data from clinical challenges were of sufficient quality and quantity, the ED01 was selected as the reference dose. In practice, the ED01 is the most stringent value that can be generated with confidence even with the datasets for which the data are most abundant. In particular it avoids low dose extrapolation, where the result is heavily dependent on the statistical model used rather than the input data (Crevel et al., 2007). The significance of the ED01 must also be appreciated. Dose distribution models describe the frequency of challenge responses associated with objective individual thresholds and presumably milder responses than incremental doses associated with the more severe reactions that are consistent with anaphylaxis, which would likely be represented by a parallel dose distribution shifted to the right. Thus, a reference dose based on the ED01 would be expected to protect at least 99\% of people allergic to the particular food from any objective reaction and minimize the probability of any serious reaction occurring in the remaining $1 \%$. Nevertheless, acknowledging that these highly sensitive individuals in the population may be avoiding commercially processed foods due to prior experiences of adverse reactions to these products in the community, the possibility of a reaction of greater severity than a mild objective reaction occurring in a very small fraction of this remaining $1 \%$ of the allergic population cannot be excluded on the basis of current knowledge.

In situations where the data were insufficient to allow estimation of the ED01, the lower 95\% confidence interval of the ED05 which would likely protect $97-99 \%$ of the affected population was selected as the reference dose. The lower 95\% confidence interval reflects the uncertainty around the ED05 estimate and is thus preferable to an arbitrary uncertainty factor directly applied to the ED05. Because the ED01 or the 95\% lower confidence intervals of the ED05 based upon human clinical data were used, additional uncertainty factors were not incorporated into the establishment of the reference doses (see Section 3.3).

The dose distributions for both discrete and cumulative doses were modelled separately using each of the three parametric models. While use of the discrete dose is the most conservative choice for risk assessment purposes, little difference was observed in ED values on this basis so the models based on both discrete and cumulative doses were considered in every case in the derivation of the reference dose, as were each of the different statistical models. Insufficient data existed to allow separate threshold dose distributions to be built for infants and children (up to the age of 18 years) versus adults except in the case of peanut. In some cases such as milk and egg, most of the available data pertained to infants and children, while in other cases (hazelnuts), the majority of the data were available for adults. Also, many studies did not report age data in sufficient detail (i.e. at the individual patient level). Thus, the data for both children and adults were combined for most allergenic foods for purely pragmatic reasons and to increase the robustness of the statistical analyses.

In the future, it would be desirable to have individual threshold data stratified by age into narrower age ranges such as exist for nutritional databases e.g. 0-3 years, 4-6 years, etc. For some foods such as milk and egg where the allergy is often outgrown, young children are likely to represent the most sensitive segment of the population. The effect of age, if any, is less certain for foods such as peanut where allergy is more persistent. As more age-specific data become available in the future, any differences in the distributions for children and adults should be considered.

For each allergenic food, Table 2 provides the total number of subjects with a clinically determined individual threshold, the number of left-censored and right-censored subjects, the number of subjects found in publications and from unpublished clinical 
records, and the clinical population (children and/or adults) where age was indicated in the publications and unpublished records. Sufficient data, i.e., enough data points to ensure that the ED value was not generated by low-dose extrapolation beyond the lowest data point (Crevel et al., 2007), were found for peanut, milk, egg, and hazelnut to allow use of the ED01 as the reference dose. Smaller amounts of individual threshold data were found for soybean, wheat, cashew, mustard, lupin, sesame seed, shrimp, celery and fish. In the case of fish, different species had also been used. For the foods with smaller available data sets, the risk assessment would be enhanced by additional data.

These data have previously been evaluated by a Scientific Expert Panel on behalf of the Allergen Bureau of Australia and New Zealand during the recent revision of the VITAL programme. The findings of that group have now been published and include more extensive assessment of the data for each of the allergenic foods (Allen et al., 2013; Taylor et al., 2014). For the most part, existing data were sufficient to allow development of reference doses at the ED01 or the 95\% lower confidence interval of the ED05. However, several data gaps and uncertainties remain.

While a reference dose of $1.0 \mathrm{mg}$ of wheat protein was established with respect to IgE-mediated wheat allergy, the question arises whether this reference dose would also be protective of coeliac patients. The Codex guideline for gluten-free products is $<20 \mathrm{ppm}$ gluten which was established based upon a no-effect level of $10 \mathrm{mg}$ of gluten per day from a 90-day intervention study (Catassi et al., 2007). On this basis, wheat-allergic consumers could safely consume $50 \mathrm{~g}$ of a gluten-free product having $20 \mathrm{ppm}$ gluten. However it is currently not clear whether certain gluten-free products may still contain small amounts of non-gluten wheat proteins and the ensuing residual risk, if any, to some people with wheat allergy.

Data on soybean thresholds were more problematic because some soy-allergic individuals have recently been identified who react to certain types of soy milk but can safely consume other forms of soy (Mittag et al., 2004). Since these individuals are not apparently allergic to all forms of soy, the data from such patients relative to thresholds were not included in the analysis. Instead, reliance was placed on individual threshold data from patients reactive to soy flour who apparently react to all forms of intact (non-hydrolyzed) soy protein. However, the possibility of several forms of soy allergy with perhaps differing threshold doses merits further investigation. With crustacean shellfish, sufficient data exist to establish a reference dose for shrimp. However, no data exist on individual thresholds for other species of crustacean shellfish such as crab and lobster. Similarly no relevant data were identified for any molluscan shellfish. Thus, uncertainties remain regarding whether the reference dose for shrimp can be also applied to crab and lobster.

Several data gaps were also identified. Insufficient data exist on celery and fish to allow establishment of a reference dose. With fish, the situation is possibly complicated by the existence of numerous fish species. Uncertainty remains regarding whether a reference dose for one species of fish would extend to all fish species. With the tree nuts, sufficient data existed on individual thresholds for hazelnut to allow establishment of a reference dose at the ED01 level. Threshold data were available from 31 cashewallergic individuals, however, the data were found only on children from one Dutch clinic so some uncertainty exists regarding whether this threshold distribution would be representative of adults and cashew-allergic patients from other geographic locations. A complete absence of data was found for most of the other tree nuts on the EU list (walnut, almond, pecan, pistachio, Brazil nut, macadamia nut). Thus, it would be premature to establish a reference dose for any tree nuts, other than for hazelnut at this time. The Allergen Bureau of Australia and New Zealand decided to use the reference dose for hazelnut ( $0.1 \mathrm{mg}$ protein) for all other nuts, while recognising the shortcomings of that strategy.

\section{Intake assessment (consumption and contamination, quality and quantity of data, differences in behaviour, quality of analytical methods)}

\subsection{Exposure assessment}

As discussed previously, risk is a function of hazard and exposure, which is therefore a critical component of risk assessment. With allergenic foods mandated to be labelled whenever used as ingredients, the relevant exposure in food allergy risk assessment is the exposure to unintended presence of allergens i.e. contamination. However, the approach described could also be adapted as a basis for labelling decisions on clinically trivial amounts of allergenic ingredients in products. Exposure assessment has two components: the level of contamination and the intake (amount and frequency) of the culprit food or foods. Both the level and frequency of contamination in a product along with the estimated product consumption (and frequency) can be used in food allergy risk assessment to generate an allergen intake distribution. Quantitative risk assessment such as probabilistic modelling (described in detail in a later section) can be used to estimate the probability of an allergic reaction occurring based on the concentration of the allergen in the product, the amount of product consumed, and the probability that an allergic person with a threshold lower than the allergen content would consume it. Accurate exposure assessment thus plays an important role in the overall risk assessment and a number of variables must be carefully considered as discussed below.

\subsection{Food intake}

Symptoms of IgE-mediated reactions to food in general appear within minutes up to a few hours after exposure. It is assumed that food allergens, if they do not cause disease, are eliminated between meals, with the prerequisite that meals are at least $2-3 \mathrm{~h}$ apart. The exposure scenario for risk assessment is therefore based on intake of the specific food during one meal or eating occasion.

Many countries collect dietary data in food surveys in a format which allows calculation of the daily intake of a particular food or food group and sometimes also intake of that food per meal in different age groups as well as the frequency of that intake. For practical reasons dietary surveys assemble the same category of food in food groups. From country to country the grouping and level of detail may vary. Bread may be a food group in one country. In another country bread may be divided into wheat bread, wheat bread with kernels, wheat bread with seeds and nuts, rye bread, rye bread with kernels or rye bread with seeds or nuts. The underlying purpose behind food intake surveys is to estimate nutrient intake and understand dietary habits e.g. intake of fruits, vegetables, red meat or soft drinks. They are also used to estimate chronic intake of various environmental contaminants (e.g. heavy metals, carcinogens, etc.). The structure of dietary data reflects these purposes and is often not optimal for food allergen risk assessment. In Europe there is only food intake data suited for food allergen risk assessment at a national level. Although many foods are produced for a pan-European market, risk assessment therefore currently needs to be done country by country. The range and type of food products sampled will therefore also differ and will depend on the specific risk assessment question, as discussed later.

National food surveys are performed on a representative sample of the population using a variety of methods, including recall and food diaries. In the latest survey from Denmark, covering the 
period 2003-2008, self-reported food allergy was mentioned by $310 / 2670(11.6 \%)$ of those surveyed. Clearly those with a food allergy should avoid foods containing as an ingredient the allergen to which they react, and this will be reflected in the survey in most cases. However, such surveys do not provide information about the consumption by allergic people of products that could contain, through cross-contact, the allergen to which they react. Although for purposes of conducting any risk assessment, it must be assumed that if a food-allergic person eats a certain food, the amount and frequency of consumption is similar to that of the general population, this is almost certain to result in under- and overestimates of the risk in many circumstances. Under-estimates would arise when food-allergic individuals substitute and consume one food more regularly than another. For instance, individuals with coeliac disease or wheat allergy following a gluten-free diet might consume more rice than the average person. If that rice were contaminated with wheat, any such individual with coeliac disease who also had a wheat allergy could be at higher risk of wheatassociated reactions from rice than the general population. Overestimates could arise if individuals with a specific food allergy actively avoided all foods perceived as "risky";, for example, milk allergic subjects avoiding all chocolate. Thus, the risk of reaction from consumption of undeclared milk in chocolate would be much less than estimated for the general population. A general assumption remains that the amount of food consumed by food-allergic individuals and its distribution will be similar to that consumed by the general population. While this may be a reasonable assumption for most food scenarios, formal proof is currently lacking.

In chemical risk assessment the mean intake per day of a food group in the whole population is often used. This figure is not very useful in food allergy risk assessment as it does not say anything about the amount of food consumed on one meal occasion. As an example the mean consumption of wheat bread per meal in the Danish adult population includes all those who do not eat bread at a meal and does not therefore correspond to the actual amount eaten and therefore the risk incurred if it is contaminated by an allergen. A more relevant figure is the mean consumption based on the persons eating bread (eaters only). The consumption may also be expressed as a percentile (e.g. the 95th percentile) intake in eaters only. In a worst case scenario the maximum intake of the food may be used. Using data from the Danish National Food Survey the mean intake per meal of wheat bread is $75 \mathrm{~g}$. The 95th percentile is $154 \mathrm{~g}$ and the maximum per meal is $355 \mathrm{~g}$. It is apparent that the measure of intake varies considerably depending on the data chosen. All the data presented are valid descriptions of wheat bread consumption, but the outcome of the risk assessment will be heavily influenced by which intake data are chosen.

Probabilistic risk assessment is not restricted to using one data point for intake (e.g. mean intake of eaters only) but can include the whole distribution of intakes across the population. If we use the bread example again it will be possible to include individual consumption data that include portion sizes and frequency of intake.

A food producer may want to know if contamination of a product with a particular amount of an allergen could pose a risk to consumers allergic to the specific contamination. Public health authorities may also want to know if a specified level of contamination would still remain acceptable if it occurred in more than one product or category of products. A scenario with contamination of more than one food with the same allergen is described in Section 5.2 below.

There has rightly been much emphasis on getting and using the best possible food challenge data as a basis on which to determine reference doses. The choice of food intake data also influences the outcome of the risk assessment and the quality of food intake data is therefore also an important consideration regarding the impact of allergens on public health.

\subsection{Level of Contamination}

The concentration of allergenic food residue (or protein from the allergenic source) can be determined either through calculation or by quantitative analysis of the ingredient or finished food product in question. Calculation of the concentration of allergenic residue can be made in instances where unintended allergen may have been included in a formulation at a consistent level (e.g. a flavour supplier changed the formulation to include a milk ingredient but failed to notify the manufacturer of the finished food). The most common approach to determining the concentration of allergenic residue is through quantitative analysis of the ingredient or finished product. Since specific proteins from the allergenic source are targeted by the immune system during IgEmediated food allergy, the analytical method used to assess the potential concentration of allergenic residue would ideally detect proteins from the allergenic source either as total protein, a certain protein fraction (e.g. casein), or a specific allergen (e.g. Ara h 1 from peanut). However, in risk assessment terms, the critical point is to be able to relate an analytical value to the human threshold data from clinical challenges as discussed below.

Immunochemical methods such as Enzyme-Linked Immunosorbent Assays (ELISA) have become the standard method used by the food industry for both qualitative and quantitative detection of allergen residues in food products or on equipment contact surfaces (Wang et al., 2010; Jackson et al., 2008). ELISAs are the favoured analytical methodology as they detect protein(s) from the allergenic source of interest, are sufficiently sensitive with detection limits in the low milligram per kilogram (ppm) range, and provide rapid assessment that can be run in the food processing facility or in a food industry laboratory (Jackson et al., 2008). Tests based on the polymerase chain reaction (PCR) are available for detection of DNA from a number of allergenic sources where ELISA methods may not be available. PCR tests do not however detect proteins from the allergenic source so their utility in food allergy risk assessment is limited (van Hengel, 2007). Advances in the use of mass spectrometry methods for the qualitative and quantitative detection of allergenic food proteins have been made over the past few years (Johnson et al., 2011). An advantage of mass spectrometry over PCR methods is that mass spectrometry methods can detect the allergenic proteins of interest and thus can provide a direct evaluation of the level of allergenic residue of concern for risk assessment purposes. The limits of detection and quantification of mass spectrometry methods are approaching the sensitivity of ELISA methods in several food matrices that have been examined and may provide a reliable confirmatory method in the foreseeable future. Other analytical methods such as the Adenosine Tri-Phosphate (ATP) test and total protein tests are utilized by food industry for routine monitoring of cleaning and sanitation but are not specific for the detection of proteins from the allergenic source of interest. While these methods are useful tools for monitoring the cleaning process, they do not provide the quantitative detection of specific proteins from the allergenic source of interest that is needed to conduct a thorough risk assessment.

ELISA methods do have their inherent differences from one commercial method to the next and these need to be fully understood and carefully considered and controlled for to ensure that the results are meaningful and can be applied to the risk assessment process. Immunochemical methods such as ELISAs utilize animal IgG antibodies that are directed against either specific allergenic proteins or, in most cases, several proteins from the allergenic source of interest (but not limited to proteins that cause IgE-mediated food allergy in humans). Interpretation of the results obtained from ELISA testing becomes a very important part of the overall allergen risk assessment process. As discussed previously, use of realistic consumption estimates for the particular product 
can then be used along with the analytical data from ELISA testing to calculate the dose per eating occasion and determine if the product contains amounts of allergenic residue at or below the reference doses for the specific allergen of interest. Converting the analytical data from ELISA methods is not always a straightforward calculation as some commercial ELISA kits report results in ppm of whole food, some in ppm protein, and others on ppm of a specific whole allergenic food. It is very important to determine the units in which the ELISA results are reported so that the proper conversions can be made. Detection of milk residue serves as a good example as there are commercial ELISA kits that detect 'total milk' (protein from both the casein and whey protein fractions of milk), caseins, and beta-lactoglobulin (BLG; from the whey fraction of milk). Some of these kits will report the results in ppm $(\mathrm{mg} / \mathrm{kg})$ non-fat dry milk (NFDM), ppm casein or ppm BLG.

Appropriate conversion factors need to be incorporated to determine the concentration in units of milk protein to correctly compare the exposure dose (in mg milk protein) to the reference dose for milk ( $0.1 \mathrm{mg}$ milk protein) which is based on the population threshold distribution of milk-allergic individuals. NFDM contains approximately $35 \%$ milk protein so if the ELISA results indicate that $10 \mathrm{ppm}$ NFDM are present in the food product, a level of $3.5 \mathrm{ppm}$ milk protein would be present. Caseins accounts for approximately $80 \%$ of milk protein so $10 \mathrm{ppm}$ casein would correspond to $12.5 \mathrm{ppm}$ milk protein. Milk contains approximately $10 \%$ BLG so a concentration of $10 \mathrm{ppm}$ BLG corresponds to $100 \mathrm{ppm}$ milk protein. Misinterpretation of the analytical results could clearly have significant effects on the overall risk assessment.

Proteins are also known to vary in terms of thermal and proteolytic stability, which can affect extraction and detection of the allergenic protein residue of interest. However inability or reduced ability to detect an allergen in an assay as a result of such changes does not necessarily translate to absence of, or reduced allergenicity. For instance, detection of BLG after thermal processing treatments such as baking has been shown to decrease likely due to the protein becoming insoluble (Downs and Taylor, 2010), but it remains to be shown through controlled challenges whether the allergenicity of the BLG has been reduced. It is always critical to validate that the ELISA will detect the allergenic residue of interest reliably, and where appropriate quantitatively, by analysing a positive control sample (a sample that is known to contain a given amount of the allergenic source of interest). As demonstrated, use of analytical methodology must be carefully considered when results are to be applied to the exposure assessment and the overall risk assessment process to ensure that a realistic estimation of risk can be made.

Probabilistic risk assessment as discussed in detail in the following section can incorporate the distribution, rather than a point estimate of the unintended allergenic residue into the risk assessment model. Analytical assessment of a number of samples taken from a batch or multiple batches of production can be used to establish a distribution of the concentration of allergenic residue that may be expected over time during a production cycle. Selecting a sufficient number of samples to obtain a representative distribution of the expected concentration of the allergenic residue is somewhat straightforward when the allergenic residue of concern is homogeneously distributed in the product of interest. Sampling becomes more challenging when the source of contamination is due to particulates which can be randomly distributed throughout the product in question. In this instance, the likelihood and size distribution of the particulate contamination, along with the dose distribution (based on the expected size distribution of the particles) can be included as input variables in the risk assessment model. Development of approaches to manage particulate contamination with food allergens is in its very early stages and has not been investigated experimentally. Validating or verifying the outputs of such models will likely prove quite challenging and will depend on the ability to develop robust sampling plans. Such a situation is analogous to that encountered in verifying microbiological standards and a first approximation would therefore be to design a sampling plan based on the approaches developed to demonstrate compliance with microbiological specifications (Codex Alimentarius Commission, 1999a; WHO-FAO, 2008).

\section{Safety and risk assessment}

\subsection{Elaboration of action levels: translating hazard characterisation to risk assessment}

The previous two subsections describe hazard characterisation and exposure assessment respectively. As previously discussed, several approaches can be used to combine these two elements of risk assessment, depending on the risk management question posed and the amount and quality of data available. This section describes how advanced techniques which take into account the totality of the available data can be used to assess the risk from the unintended presence of food allergens. It considers in particular how these data may be combined with information on food intake and unintended allergen concentration to derive action levels, which are quantitative benchmarks for allergen management. An action level in this context is the concentration in a product of protein from an unintended allergenic constituent which would deliver a dose of allergen equivalent to the accepted reference dose. Operationally, action levels can serve to define the concentrations of allergen below which a precautionary warning would be deemed unnecessary because the risk posed by the presence of unintended allergen was below the accepted level of risk as defined by the relevant reference dose. In contrast, concentrations in excess of the action level would trigger additional risk reduction measures or, if not feasible, a precautionary warning.

Action levels can be derived from reference doses and food intake and contamination data in several ways. Two approaches, deterministic and probabilistic are illustrated in Fig. 1 below.

The deterministic approach, illustrated by method 1 in the figure describes a simple arithmetical method and can be used when no or limited data are available on the consumption of the food of interest or its distribution. It is also most practical for elaboration of action levels by industry.

Action levels can most easily be calculated from an ED-value chosen as a reference dose and an assumed intake (portion size) by the following formula:

[EDp (in mg)/Intake (in $\mathrm{kg}$ )] = action level (in $\mathrm{mg} / \mathrm{kg}$ or ppm)

EDp: dose calculated to elicit an allergic reaction in p\% of allergic individuals.

Clearly when elaborating action levels through combination of a chosen food consumption figure and an ED-value, the choice of the appropriate consumption figure is critical. This can be illustrated by the following example.

A reference dose of $0.2 \mathrm{mg}$ protein was elaborated for peanut based on a proposed maximum accepted risk of $1 \%$ (see Table 2 and Section 5.2). In the Netherlands, one slice of bread is considered the portion size for sliced brown wheat bread, but the mean consumption per meal occasion is $140 \mathrm{~g}$, while the p95 intake for male users of brown wheat bread is $210 \mathrm{~g}$. The action level based on a $35 \mathrm{~g}$ portion size is $5.7 \mathrm{ppm}$, whereas the action level corresponding to the p95 intake of $210 \mathrm{~g}$ is only $1 \mathrm{ppm}$. Underestimating consumption thus results in excessively high action levels and associated higher actual risks. However, at the same time, it should be remembered that the elaboration of reference doses embodies several worst case assumptions and choices. It does not benefit 


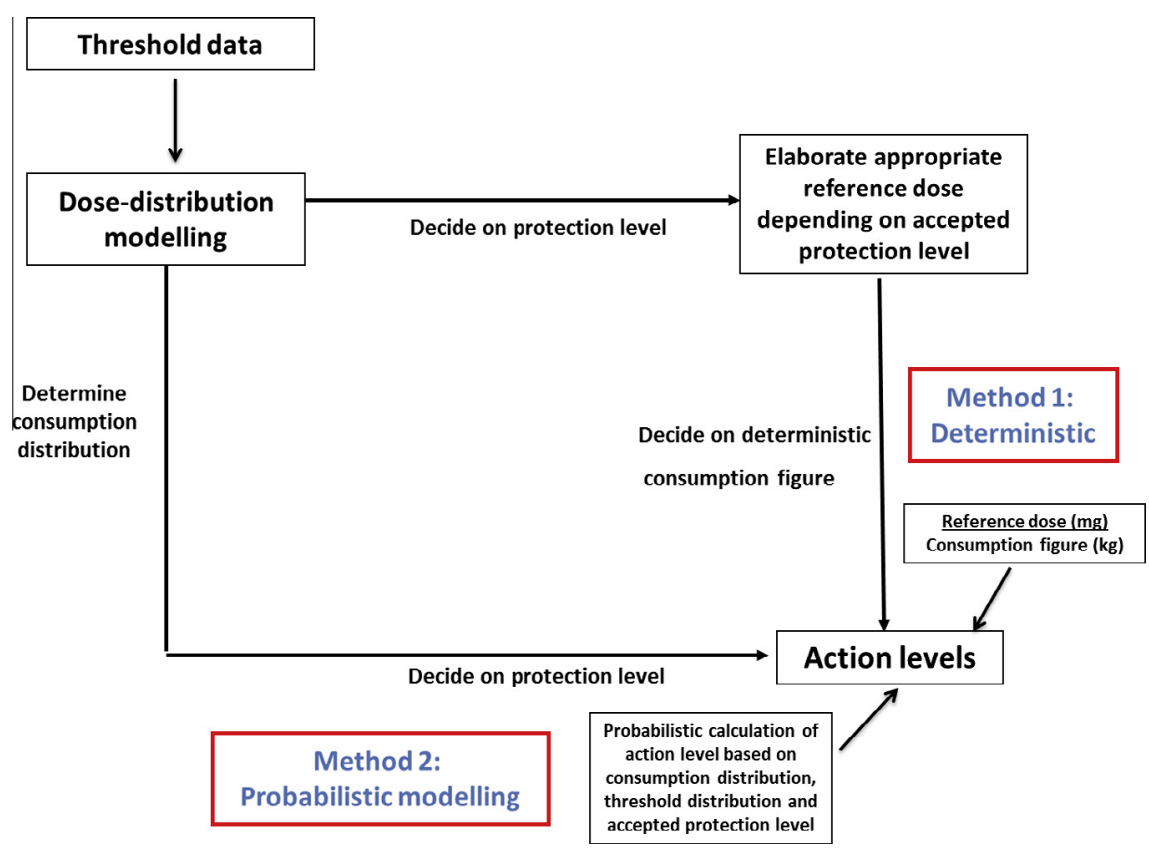

Fig. 1. Elaboration of concentration action levels for "may contain" labelling.

any stakeholder if too much conservatism is built in, as this could potentially lead to an unnecessarily high proportion of products carrying a precautionary warning on the label and the risk of a resulting loss of credibility (Cochrane et al., 2013). Of course, the deterministic approach can be used as a basis for risk assessment, although it cannot yield a quantitative output, as discussed elsewhere (Madsen et al., 2009).

Probabilistic modelling (Method 2) can also be used to derive action levels using food intake and minimum eliciting dose distributions, as well as a certain accepted residual risk level as a starting point. Probabilistic risk assessment modelling is illustrated conceptually in Fig. 2 below.

In practice the program repeatedly samples the input distributions picking a value from each at random and builds a distribution representing the probability of an allergic reaction given the values and distributions of the specified variables. Probabilistic modelling inherently accounts for the some of the uncertainties associated with the input variables and reflects those in the probability distribution for the output. It is important to note, however, that probabilistic modelling cannot take into account factors such as

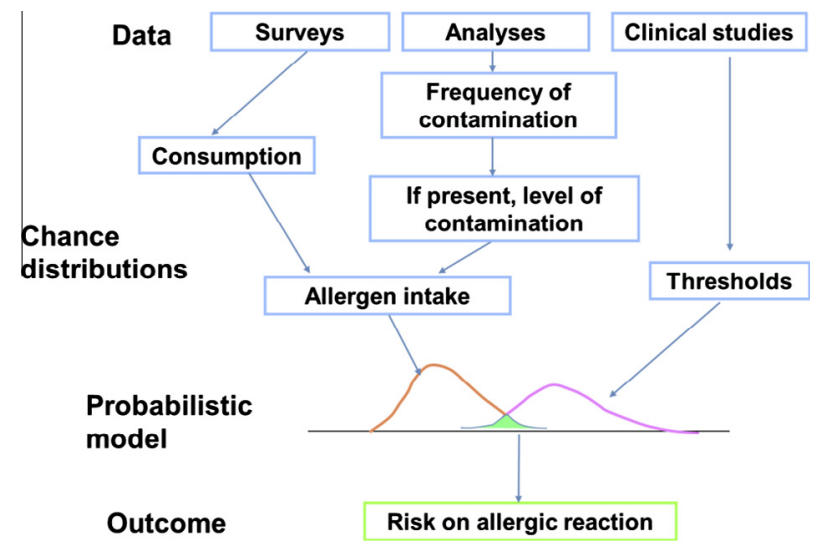

Fig. 2. Probabilistic risk assessment (adapted from Spanjersberg et al., 2007). systematic bias in the selection of the challenge population, unless they can be quantified. This approach is generally considered the most appropriate approach for population risk assessment goals (Spanjersberg et al., 2007, 2010; Madsen et al., 2009; Kruizinga et al., 2008). Since its initial application to food allergen risk assessment, it has been developed further with the introduction of Bayesian inference of input variables (Rimbaud et al., 2010, 2013), coupling it with second-order Monte Carlo (MC2) simulations to separately propagate the variability and uncertainty associated with each of these variables.

Probabilistic risk assessment can also be used for instance to validate elaborated action levels (for instance: have I chosen an adequate consumption figure so that the residual risk at the action level is $1 \%$ or below?) or for more precise quantification of the risk of certain contamination and consumption scenarios. Another application of probabilistic risk assessment is to quantify the effect of adherence to a certain risk management regime or to investigate the public health benefit of a more stringent cleaning regime.

\subsection{Validation of action levels through probabilistic risk assessment}

A critical question, particularly for regulators, concerns the risk posed by consumption of more than one product contaminated with the same allergen, and therefore to what extent such a possibility needs to be considered in deciding on reference doses. As described above, probabilistic modelling can be applied to estimate the number of allergic individuals or the proportion of an (allergic) population expected to suffer an allergic reaction due to a certain concentration distribution of an allergen in food products (the residual risk). As such, the approach is very suitable for assessing whether the risk of allergic reactions for products containing allergenic residues at the action levels indeed remains around or below the level of risk accepted. The expert group has used probabilistic risk assessment to verify the conclusions about action levels in relation to amount consumed in the peanut in bread example cited earlier with the same result as the deterministic calculations. However, probabilistic modelling scores over such simple deterministic calculations in the case of more complex scenarios such as assessing the impact of the combined consumption during one meal of 2 
Table 3

Dutch Food Consumption Survey data for selected food.

\begin{tabular}{|c|c|c|c|}
\hline \multicolumn{4}{|c|}{ Consumption of products in the Netherlands (excluding zero) } \\
\hline & $\%$ Users of product in population & Mean (g/meal occasion) & P95 (g/meal occasion) \\
\hline Mashed potato powder & 2.3 & 5.1 & 9.4 \\
\hline Sauce from a package, including creamy sauces & 15.6 & 72 & 200 \\
\hline Chocolate spread milk & 3.3 & 43.1 & 180 \\
\hline Bread brown - wheat & 62.8 & 118 & 210 \\
\hline
\end{tabular}

products that are both contaminated with the same allergen but at different frequencies.

Two independent food intake scenarios were assessed: (1) a meal with mashed potato and a sauce and (2) a meal with bread and a chocolate spread. Each food intake scenario was assessed for the risk arising from unintended peanut presence. The analyses were conducted using food intake data from the US, Denmark and the Netherlands. The outcomes of the impact analyses were similar, and for brevity, only Dutch data are presented and discussed here.

The following consumption data were derived from the Dutch Food Consumption survey (Hulshof et al., 2003) (see Table 3).

Action levels were calculated based on both the mean and the p95 consumption data. The reference dose for peanut based on the ED01 $(0.2 \mathrm{mg})$ as given in Section 3.3 was used to determine the action level. This resulted in the action levels below (Table 4).

Probabilistic risk assessment was done as described before (Spanjersberg et al., 2007, 2010). All scenarios were run to calculate the risk in the population of (peanut)-allergic users of the product (i.e. prevalence of peanut allergy is not a variable). For simplicity, we used point estimates to illustrate the scenarios where a limited proportion of the products were contaminated, although in practice, this would be a distribution with an associated uncertainty and variability. We did not have adequate data to describe the occurrence of peanut cross-contact for the specific products in question. Were such data available, it would be possible to perform a complete probabilistic analysis analogous to that described by Rimbaud et al. (2013) for peanut contamination of confectionery products in France. Such an approach could also be used to derive action levels which would assure compliance with defined reference doses according to specified criteria (e.g. 95\% assurance that the reference dose would be met).

For each product (combination) the risk was analysed in the following situations (contamination was assumed to be at the action level):

- Product 1, all products contaminated with the allergen.

- Product 2, all products contaminated with the allergen.

- Product 1, 10\% of the products contaminated with the allergen.

- Product 2, $10 \%$ of the products contaminated with the allergen.

- Product 1 and product 2 at the same eating occasion with each of the products having a chance of $10 \%$ of being contaminated.

- Product 1 and product 2 at the same eating occasion with each of the products having a chance of $100 \%$ of being contaminated.

Table 4

Action levels based on reference doses and calculated for mean and P95 consumption.

\begin{tabular}{lll}
\hline & $\begin{array}{l}\text { Action level based on } \\
\text { mean consumption } \\
(\mathrm{mg} / \mathrm{kg})\end{array}$ & $\begin{array}{l}\text { Action level based on P95 } \\
\text { consumption }(\mathrm{mg} / \mathrm{kg})\end{array}$ \\
\hline $\begin{array}{ll}\text { Mashed potato } \\
\text { powder }\end{array}$ & 39.2 & 21.3 \\
$\begin{array}{l}\text { Sauce } \\
\text { Chocolate spread }\end{array}$ & 2.8 & 1 \\
Bread brown - & 4.6 & 1.1 \\
wheat & 1.7 & 1 \\
\hline
\end{tabular}

The threshold distributions for the probabilistic modelling were based on the work performed by the VITAL Scientific Expert Panel and described in subSection 3.3, although only the dose distributions for adults were used. As the goal of the assessment was to investigate whether the risks from combined exposure scenarios would be significantly higher than the targeted residual risk of $1 \%$, risk assessment based on action levels derived on the basis of mean consumption can be considered as a worst case for this analysis as they are the higher ones. Only those results are shown. Finally, only the log logistic threshold distributions are shown as they predicted the highest risk. The risk assessment results are summarized in the Tables $5 \mathrm{a}$ and $5 \mathrm{~b}$ below.

The results demonstrate clearly that only where all food products (so $100 \%$ of the batches produced) are assumed to be contaminated with peanut at the action level, is the residual risk slightly higher than the $1 \%$ accepted residual risk. Where it is assumed that only $10 \%$ of the batches are contaminated, fewer than $1 \%$ of peanut-allergic consumers are predicted to be at risk of a reaction, even if we assume that for the $10 \%$ of the batch that is contaminated the contamination at the maximum level (i.e. at the action level), which still is a unrealistic worst case assumption. All other risk estimates remained below the targeted $1 \%$ maximal residual risk.

Table 5a

Residual risk from consumption of products contaminated with the same unintended allergen (Illustration with mashed potato and sauce).

\begin{tabular}{lll}
\hline Contaminated products & $\begin{array}{l}\text { Proportion of } \\
\text { products } \\
\text { contaminated }(\%)\end{array}$ & $\begin{array}{l}\text { Risk assessment } \\
\text { outcome } \\
(\% \text { residual risk) }\end{array}$ \\
\hline Mashed potato & 100 & 0.82 \\
Mashed potato & 10 & 0.08 \\
Sauce & 100 & 0.83 \\
Sauce & 10 & 0.08 \\
Mashed potato with & 100 & 1.32 \\
$\quad \begin{array}{l}\text { sauce } \\
\text { Mashed potato with }\end{array}$ & 10 & 0.13 \\
$\quad$ sauce &
\end{tabular}

a Proportion of peanut-allergic people eating the product combinations expected to react.

Table 5b

Residual risk from consumption of products contaminated with the same unintended allergen (Illustration with bread and chocolate spread).

\begin{tabular}{lll}
\hline Contaminated products & $\begin{array}{l}\text { Proportion of products } \\
\text { contaminated (\%) }\end{array}$ & $\begin{array}{l}\text { Risk assessment } \\
\text { outcome } \\
\text { (\% residual risk) }\end{array}$ \\
\hline Bread & 100 & 0.83 \\
Bread & 10 & 0.08 \\
Chocolate spread & 100 & 0.72 \\
Chocolate spread & 10 & 0.07 \\
Bread with chocolate & 100 & 1.26 \\
$\quad$ spread & 10 & 0.13 \\
Bread with chocolate & & \\
$\quad$ spread & & \\
\hline
\end{tabular}


From these scenario analyses it can be safely concluded that the risk from combined consumption of food products incidentally contaminated with the same allergen will remain at or below the maximum targeted residual risk ( $1 \%$ in this scenario analyses). Combined consumption of food products contaminated with a same allergen thus poses no significant additional health risk.

\section{Conclusions}

Risk assessment is at the heart of managing the risk from allergens in the interests of all stakeholders. In recent years it has become generally accepted that the principles and methodologies developed for toxicological risk assessment can be applied to food allergens as contaminants. While allergy being an immune response means that risk can be considered from the perspective of initiation of an immune response (sensitisation) as well as elicitation (reaction), the specific risk assessment for food allergens question focusses on the ability of (generally) low levels of allergenic residues in manufactured foods to provoke reactions in sensitised individuals. This risk can be quantified on the basis of data from blinded diagnostic food challenges. The data used may have limitations related to both the selection of participants and the investigation of reactivity. However, these limitations are outweighed by the generation and availability of more robust data and modelling approaches that do not rely upon low-dose extrapolations from dose-distributions derived from clinical challenges with their inherent uncertainties.

Understanding how to apply this risk assessment approach in the food allergen stakeholder community is still challenging. Good quality and amounts of data have become available for a range of regulated allergens from food challenge studies, which have also revealed that the range of MEDs spans at least 5-6 orders of magnitude, making it difficult to derive a usable population NOAEL and spurring the development of statistical models to analyse the data meaningfully. Exposure assessment is a critical component of risk assessment, but has not received as much attention. Exposure estimates require not only good analytical tools to quantify allergens, but also an understanding of the food consumption habits of people with food allergies. Nevertheless general food consumption data together with dose distribution data have proved particularly valuable in combination with probabilistic risk assessment techniques, which are able to integrate the uncertainties around many of the inputs into a single one relating to the risk outcome. Application of these models has provided useful insights which are not only driving further refinements, but also generating testable hypotheses. Results of model predictions have also thrown into sharp focus the framing of the risk management questions, in particular those relating to the type of reaction that risk management seeks to avoid.

\section{Declaration of interest}

A. Baka is employed by ILSI Europe.

S. Luccioli did not receive funding from ILSI Europe and the findings and conclusions in this article should not be construed to represent US FDA determination or policy.

\section{Conflict of Interest}

The authors declare that there are no conflicts of interest.

\section{Transparency Document}

The Transparency document associated with this article can be found in the online version.

\section{Acknowledgements}

This work was conducted by an expert group of the European branch of the International Life Sciences Institute (ILSI Europe). The expert group received funding from the ILSI Europe Food Allergy Task Force. Industry members of this task force are listed on the ILSI Europe website at www.ilsi.eu.For further information about ILSI Europe, please email info@ilsieurope.be or call +32 2 7710014 . The opinions expressed herein and the conclusions of this publication are those of the authors and do not necessarily represent the views of ILSI Europe nor those of its member companies.

The authors wish to thank the members of the Expert Group: Dr. Yong Joo Chung, Dr. Anke Ehlers, Dr. Marcel Feys, Dr. Corinne Herouet-Guicheney, Dr. Jonathan Hourihane, Prof Marek Kowalski, Prof Alfonso Lampen, Dr. Hubert Noteborn, Prof Nikolaos Papadopoulos, Dr. Fabrice Peladan, Mr. Stefan Ronsmans, Mr. Frans Timmermans and Prof Margitta Worm for their invaluable contribution to this work through their enthusiastic and generous participation.

\section{References}

Allen, K.J., Remington, B.C., Baumert, J.L., Crevel, R.W., Houben, G.F., Brooke-Taylor, S., Kruizinga, A.G., Taylor, S.L., 2013. Allergen reference doses for precautionary labeling (VITAL 2.0): clinical implications. J.Allergy Clin.Immunol.. http:// dx.doi.org/10.1016/j.jaci.2013.06.042 (Epub ahead of print).

Ballmer-Weber, B.K., Holzhauser, T., Scibilia, J., Mittag, D., Zisa, G., Ortolani, C., Oesterballe, M., Poulsen, L.K., Vieths, S., Bindslev-Jensen, C., 2007. Clinical characteristics of soybean allergy in Europe: a double-blind, placebo-controlled food challenge study. J. Allergy Clin. Immunol. 119, 1489-1496.

Bindslev-Jensen, C., Briggs, D., Osterballe, M., 2002. Can we determine a threshold level for allergenic foods by statistical analysis of published data in the literature? Allergy 57, 741-746.

Bindslev-Jensen, C., Ballmer-Weber, B.K., Bengtsson, U., Blanco, C., Ebner, C., Hourihane, J., Knulst, A.C., Moneret-Vautrin, D.A., Nekam, K., Niggemann, B., Osterballe, M., Ortolani, C., Ring, J., Schnopp, C., Werfel, T., 2004. Standardization of food challenges in patients with immediate reactions to foods-position paper from the European Academy of Allergology and Clinical Immunology. Allergy $59,690-697$.

Bjorksten, B., Crevel, R., Hischenhuber, C., Lovik, M., Samuels, F., Strobel, S., Taylor, S.L., Wal, J.M., Ward, R., 2008. Criteria for identifying allergenic foods of public health importance. Regul. Toxicol. Pharmacol. 51, 42-52.

Bock, S.A., Sampson, H.A., Atkins, F.M., Zeiger, R.S., Lehrer, S., Sachs, M., Bush, R.K., Metcalfe, D.D., 1988. Double-blind, placebo-controlled food challenge (DBPCFC) as an office procedure: a manual. J. Allergy Clin. Immunol. 82, 986-997.

Catassi, C., Fabiani, E., Iacono, G., D’Agate, C., Francavilla, R., Biagi, F., Volta, U., Accomando, S., Picarelli, A., De, V., I, Pianelli, G., Gesuita, R., Carle, F., Mandolesi, A., Bearzi, I., Fasano, A., 2007. A prospective, double-blind, placebo-controlled trial to establish a safe gluten threshold for patients with celiac disease. Am. J. Clin. Nutr. 85, 160-166.

Clark, A.T., Islam, S., King, Y., Deighton, J., Anagnostou, K., Ewan, P.W., 2009. Successful oral tolerance induction in severe peanut allergy. Allergy 64, 1218 1220.

Cochrane, S.A., Salt, L.J., Wantling, E., Rogers, A., Coutts, J., Ballmer-Weber, B.K., Fritsche, P., Fernandez-Rivas, M., Reig, I., Knulst, A., Le, T.M., Asero, R., Beyer, K., Golding, M., Crevel, R., Clare Mills, E.N., Mackie, A.R., 2012. Development of a standardized low-dose double-blind placebo-controlled challenge vehicle for the EuroPrevall project. Allergy 67, 107-113.

Cochrane, S.A., Gowland, M.H., Sheffield, D., Crevel, R.W.R., 2013. Characteristics and purchasing behaviours of food-allergic consumers and those who buy food for them in Great Britain. Clin. Trans. Allergy 3, 31.

Codex Alimentarius Commission, 1999. Codex General Standard for the Labelling of Pre-Packaged Foods. FAO/WHO.

Codex Alimentarius Commission, 1999a. Principles and guidelines for the conduct of microbiological risk assessment. CAC/GL-30 Amendment 2012, FAO-WHO.

Codex Alimentarius, 1999b. Recommended International Code Of Practice: General Principles Of Food Hygiene. CAC/RCP 1-1969, Rev. 3 (1997), Amended 1999.

Crevel, R.W., Briggs, D., Hefle, S.L., Knulst, A.C., Taylor, S.L., 2007. Hazard characterisation in food allergen risk assessment: the application of statistical approaches and the use of clinical data. Food Chem. Toxicol. 45, 691-701.

Crevel, R.W., Ballmer-Weber, B.K., Holzhauser, T., Hourihane, J.O., Knulst, A.C. Mackie, A.R., Timmermans, F., Taylor, S.L., 2008. Thresholds for food allergens and their value to different stakeholders. Allergy 63, 597-609.

Downs, M.L., Taylor, S.L., 2010. Effects of thermal processing on the enzyme-linked immunosorbent assay (ELISA): detection of milk residues in a model food matrix. J. Agr. Food Chem. 58, 10085-10091. http://dx.doi.org/10.1021/ jf101718f. 
EFSA, 2004. Opinion of the Scientific Panel on Dietetic Products, Nutrition and Allergies on a request from the Commissiion relating to the evaluation of allergenic foods for labelling purposes. EFSA J. 32, 1-197.

Gendel, S.M., 2012. Comparison of international food allergen labeling regulations. Regul. Toxicol. Pharmacol. 63, 279-285.

Gern, J.E., Yang, E., Evrard, H.M., Sampson, H.A., 1991. Allergic reactions to milkcontaminated "nondairy" products. N. Engl. J. Med. 324, 976-979.

Grimshaw, K.E., King, R.M., Nordlee, J.A., Hefle, S.L., Warner, J.O., Hourihane, J.O., 2003. Presentation of allergen in different food preparations affects the nature of the allergic reaction - a case series. Clin. Exp. Allergy 33, 15811585 .

Hefle, S.L., Nordlee, J.A., Taylor, S.L., 1996. Allergenic foods. Crit. Rev. Food Sci. Nutr. 36 (Suppl), S69-S89.

Hourihane, J.O., Bedwani, S.J., Dean, T.P., Warner, J.O., 1997a. Randomised, double blind, crossover challenge study of allergenicity of peanut oils in subjects allergic to peanuts. BMJ 314, 1084-1088.

Hourihane, J.O.B., Kilburn, S.A., Nordlee, J.A., Hefle, S.L., Taylor, S.L., Warner, J.O., 1997b. An evaluation of the sensitivity of subjects with peanut allergy to very low doses of peanut protein: a randomized, double-blind, placebo-controlled food challenge study. J. Allergy Clin. Immunol. 100, 596-600.

Hulshof, K.F., Brussaard, J.H., Kruizinga, A.G., Telman, J., Lowik, M.R., 2003. Socioeconomic status, dietary intake and $10 \mathrm{y}$ trends: the Dutch National Food Consumption Survey. Eur. J. Clin. Nutr. 57, 128-137.

Jackson, L.S., Al-Taher, F.M., Moorman, M., DeVries, J.W., Tippett, R., Swanson, K.M., Fu, T.J., Salter, R., Dunaif, G., Estes, S., Albillos, S., Gendel, S.M., 2008. Cleaning and other control and validation strategies to prevent allergen cross-contact in food-processing operations. J. Food Prot. 71, 445-458.

Johnson, P.E., Baumgartner, S., Aldick, T., Bessant, C., Giosafatto, V., Heick, J., Mamone, G., O'Connor, G., Poms, R., Popping, B., Reuter, A., Ulberth, F., Watson, A., Monaci, L., Mills, E.N., 2011. Current perspectives and recommendations for the development of mass spectrometry methods for the determination of allergens in foods. J. AOAC Int. 94, 1026-1033.

Kruizinga, A.G., Briggs, D. Crevel, R.W., Knulst, A.C. van den Bosch, L.M., Houben, G.F., 2008. Probabilistic risk assessment model for allergens in food: sensitivity analysis of the minimum eliciting dose and food consumption. Food Chem. Toxicol. 46, 1437-1443.

Lemon-Mule, H., Sampson, H.A., Sicherer, S.H., Shreffler, W.G., Noone, S., NowakWegrzyn, A., 2008. Immunologic changes in children with egg allergy ingesting extensively heated egg. J. Allergy Clin. Immunol. 122, 977-983.

Madsen, C.B., Hattersley, S., Buck, J., Gendel, S.M., Houben, G.F., Hourihane, J.O., Mackie, A., Mills, E.N., Norhede, P., Taylor, S.L., Crevel, R.W., 2009. Approaches to risk assessment in food allergy: report from a workshop "developing a framework for assessing the risk from allergenic foods". Food Chem. Toxicol. 47, 480-489.

Malmheden-Yman, I., 2004. Detection of inadequate labelling and contamination as causes of allergic reactions to food. Acta Aliment. 33, 347-357.

Mittag, D., Vieths, S., Vogel, L., Becker, W.M., Rihs, H.P., Helbling, A., Wuthrich, B., Ballmer-Weber, B.K., 2004. Soybean allergy in patients allergic to birch pollen: clinical investigation and molecular characterization of allergens. J. Allergy Clin. Immunol. 113, 148-154.

Nguyen-Grosjean, V.M., 2013. Peanut allergy in France: preliminary results of the MIRABEL pilot study. Clin. Trans. Allergy 3 (Suppl 3), P131 (Poster) <http:// www.ctajournal.com/content/3/S3/P131>.

Nowak-Wegrzyn, A., Bloom, K.A., Sicherer, S.H., Shreffler, W.G., Noone, S., Wanich, N., Sampson, H.A., 2008. Tolerance to extensively heated milk in children with cow's milk allergy. J. Allergy Clin. Immunol. 122 (342-7), 347.
Rimbaud, L., Héraud, F., La Vieille, S., Leblanc, J.C., Crépet, A., 2010. Quantitative risk assessment related to -adventitious allergens in food: a probabilistic model applied to peanut in chocolate. Risk Anal. 30 (1), 7-19.

Rimbaud, L., Héraud, H., La Vieille, S., Leblanc, J.Ch., Crépet, A., 2013. Quantitative risk assessment relating to inadvertent presence of peanut allergens in various foodstuffs. Int. Food Risk Anal. J. 3, 4. http://dx.doi.org/10.5772/56646.

Rolinck-Werninghaus, C., Niggemann, B., Grabenhenrich, L., Wahn, U., Beyer, K. 2012. Outcome of oral food challenges in children in relation to symptomeliciting allergen dose and allergen-specific IgE. Allergy 67, 951-957.

Sampson, H.A., 1996. Epidemiology of food allergy. Pediatr. Allergy Immunol. 7, 42 50.

Sicherer, S.H., Morrow, E.H., Sampson, H.A., 2000. Dose-response in double-blind, placebo-controlled oral food challenges in children with atopic dermatitis. J. Allergy Clin. Immunol. 105, 582-586.

Skripak, J.M., Nash, S.D., Rowley, H., Brereton, N.H., Oh, S., Hamilton, R.G., Matsui, E.C., Burks, A.W. Wood, R.A., 2008. A randomized, double-blind, placebocontrolled study of milk oral immunotherapy for cow's milk allergy. J. Allergy Clin. Immunol. 122, 1154-1160.

Spanjersberg, M.Q., Kruizinga, A.G., Rennen, M.A., Houben, G.F., 2007. Risk assessment and food allergy: the probabilistic model applied to allergens. Food Chem. Toxicol. 45, 49-54.

Spanjersberg, M.Q., Knulst, A.C., Kruizinga, A.G., Van, D.G., Houben, G.F., 2010. Concentrations of undeclared allergens in food products can reach levels that are relevant for public health. Food Addit. Contam. A. Chem. Anal. Control Expo. Risk Assess 27, 169-174.

Taylor, S.L., Hefle, S.L., Bindslev-Jensen, C., Bock, S.A., Burks Jr. A.W. Christie, L, Hill, D.J., Host, A., Hourihane, J.O., Lack, G., Metcalfe, D.D., Moneret-Vautrin, D.A., Vadas, P.A., Rance, F., Skrypec, D.J., Trautman, T.A., Yman, I.M., Zeiger, R.S., 2002 Factors affecting the determination of threshold doses for allergenic foods: how much is too much? J. Allergy Clin. Immunol. 109, 24-30.

Taylor, S.L., Hefle, S.L., Bindslev-Jensen, C., Atkins, F.M., Andre, C., BruijnzeelKoomen, C., Burks, A.W., Bush, R.K., Ebisawa, M., Eigenmann, P.A., Host, A. Hourihane, J.O., Isolauri, E., Hill, D.J., Knulst, A., Lack, G., Sampson, H.A., Moneret-Vautrin, D.A., Rance, F., Vadas, P.A., Yunginger, J.W., Zeiger, R.S., Salminen, J.W., Madsen, C., Abbott, P., 2004. A consensus protocol for the determination of the threshold doses for allergenic foods: how much is too much? Clin Exp. Allergy 34, 689-695.

Taylor, S.L., Crevel, R.W., Sheffield, D., Kabourek, J., Baumert, J., 2009. Threshold dose for peanut: risk characterization based upon published results from challenges of peanut-allergic individuals. Food Chem. Toxicol. 47, 1198-1204.

Taylor, S.L., Moneret-Vautrin, D.A., Crevel, R.W., Sheffield, D., Morisset, M., Dumont P., Remington, B.C., Baumert, J.L., 2010. Threshold dose for peanut: risk characterization based upon diagnostic oral challenge of a series of 286 peanut-allergic individuals. Food Chem. Toxicol. 48, 814-819.

Taylor, S.L., Baumert, J.L., Kruizinga, A.G., Remington, B.C., Crevel, R.W.R., Brooke Taylor, S., Allen, K.J., The Allergen Bureau of Australia \& New Zealand, Houben, G., 2014. Establishment of reference doses for residues of allergenic foods: Report of the VITAL Expert Panel. Food Chem. Toxicol. 63, 9-17.

Threshold Working Group, 2008 Approaches to establish thresholds for major food allergens and for gluten in foods. 71 ed., pp. 1043-1088.

van Hengel, A.J., 2007. Food allergen detection methods and the challenge to protect food-allergic consumers. Anal. Bioanal. Chem. 389, 111-118.

Wang, X., Young, O.A., Karl, D.P., 2010. Evaluation of cleaning procedures for allergen control in a food industry environment. J. Food Sci. 75, T149-T155.

WHO-FAO, 2008. Exposure assessment of microbiological hazards in food. Microbiological Risk Assessment Series 7. Guidelines. 